\title{
The angiotensin antagonist Losartan shifts social reward motivation and punishment feedback sensitivity via modulating midbrain-striato-frontal circuits
}

\author{
Xinqi Zhou ${ }^{1}$, Ting $\mathrm{Xu}^{1}$, Yixu Zeng ${ }^{1}$, Ran Zhang ${ }^{1}$, Ziyu Qi ${ }^{1}$, Weihua Zhao ${ }^{1}$, Keith M \\ Kendrick $^{1}$, Benjamin Becker ${ }^{1 *}$
}

${ }^{1}$ The Clinical Hospital of Chengdu Brain Science Institute, MOE Key Laboratory for Neuroinformation, University of Electronic Science and Technology of China,

Chengdu, China

\section{*corresponding author}

Benjamin Becker, $\mathrm{PhD}$

Center for Information in Medicine

University of Electronic Science and Technology of China

Chengdu 611731, China

Tel.: +862861830811

Mail: ben_becker@gmx.de 


\begin{abstract}
Social deficits and dysregulations in dopaminergic midbrain-striato-frontal circuits represent transdiagnostic symptoms across psychiatric disorders. Animal models suggest that modulating interactions between the dopamine and renin-angiotensin system with the angiotensin receptor antagonist Losartan (LT) can modulate learning and reward-related processes. We have therefore determined the behavioral and neural effects of LT on social reward and punishment processing in humans. A preregistered randomized double-blind placebo-controlled between-subject pharmacological design was combined with a social incentive delay fMRI paradigm during which subjects could avoid social punishment or gain social reward. Healthy volunteers received a single-dose of LT $(50 \mathrm{mg}, \mathrm{n}=43)$ or placebo $(n=44)$. Reaction times and emotional ratings served as behavioral outcomes, on the neural level activation, connectivity and social feedback prediction errors were modelled. Relative to placebo, LT switched reaction times and arousal away from prioritizing punishment towards social reward. On the neural level the LT-enhanced motivational salience of social rewards was accompanied by stronger ventral striatum-prefrontal connectivity during reward anticipation and attenuated activity in the ventral tegmental area (VTA) and associated connectivity with the bilateral insula in response to punishment during the outcome phase. Computational modelling further revealed an LT-enhanced social reward prediction error signal in VTA and dorsal striatum. LT shifted motivational and emotional salience away from social punishment towards social reward via modulating distinct core nodes of the midbrain-striato-frontal circuits. The findings document a modulatory role of the renin-angiotensin system in these circuits and associated social processes, suggesting a promising treatment target to alleviate social dysregulations.
\end{abstract}

Keywords Losartan, Social reward, Dopamine, Prediction error, Striatum, Ventral tegmental area, Angiotensin-renin system 


\section{Introduction}

Adaptive processing of social feedback is vital for interpersonal functioning and mental health. Dysregulations in this domain and its underlying neural processes contribute to the development and maintenance of major psychiatric disoders including depression (1-3), social anxiety disorder (4), post-traumatic stress disorder (PTSD) (5), addiction (6), autism spectrum disorder (7, 8), and schizophrenia (9). While these disorders cause tremendous individual suffering and socio-economic costs the development of novel treatments that target social deficits based on the underlying neurobiological dysregulations remains challenging $(10,11)$.

The Research Domain Criteria (RDoC) framework aims to facilitate novel treatment development by conceptualizing psychiatric disorders from basic dimensions of functioning, including the domains of social communication as well as reward/loss evaluation (12). Together these domains may represent a transdiagnostic treatment target with the potential to improve social functioning. Dysregulations in midbrain-striato-prefrontal circuits have been increasingly established as a core pathogenic mechanism across psychiatric disorders (3, 13-16). Findings from human imaging studies suggest that this circuitry overlaps with that involved in social reward and punishment processing $(17,18)$ and animal models indicate that neurochemical signalling in this circuitry critically regulates social reward and punishment (19-21). Dopamine (DA) and its interactions with other neurotransmitter systems such as oxytocin play an important role in modulating social reward and punishment in these cicruits $(5,22)$, however, direct pharmacological modulation of these systems commonly results in negative side effects or highly context-dependent effects, respectively, which critically impede the clinical utility of these approaches $(11,23$, 24).

Recent pharmacological studies in healthy humans have demonstrated that targeting the renin-angiotensin system (RAS) via the angiotensin II type 1 receptor (AT1R) antagonist losartan (LT, an approved treatment for hypertension) can modulate reward and threat processing as well as learning and memory in the absence of negative side effects (25-29). Earlier animal models suggest an interaction between the RAS and the central DA system, including a dense expression of RAS receptors in midbrain-striato-prefrontal circuits (30) and functionally significant angiotensin II receptors located presynaptically on dopaminergic neurons $(31,32)$. LT induced concentration-dependent inhibition of dopamine release via inactivation of AT1R (33), but also enhanced dopamine D1 receptor (D1R) signaling which may contribute to both its effects on hypertension (34) and reward-related processes $(35,36)$. Additionally, optogenetic inhibition/activation of ventral tegmental area (VTA) dopaminergic neurons - which exhibit dense AT1R expression - revealed that cueevoked DA release accurately encodes reward prediction errors $(37,38)$, thus supporting behavioral adaptation and associative learning $(39,40)$. Together, the available evidence suggests that targeting the RAS via LT may represent a promising candidate to modulate neural processing in midbrain-striatal-prefrontal circuits which critically mediate flexible behavioral adaption in the domains of feedback-dependent learning as well as earlier stages of social and non-social reward processing $(17,18$, 
22, 41, 42). Initial evidence for the functional and behavioral relevance of this strategy in humans comes from a recent study that demonstrated that a single dose of 50mg LT can modulate feedback-dependent learning in healthy individuals such that LT enhanced the difference between loss and reward feedback learning rates and suppressed loss learning rates (29). Determining behavioral and neural effects on earlier stages of reward and punishment processing in social contexts will facilitate a translational neuroimaging approach which facilitates both translation from animal models, indicating that RAS-DA interactions modulate reward-related processing in these cicruits $(19,20,35,36)$, and translation into therapeutic application in populations with social deficits.

Reward-related neural responses in this circuitry encompass anticipatory and consummatory signals, closely linked to brain activation in the midbrain-striatalfrontal circuit, particularly the VTA, striatum, and frontal cortex $(17,18,22)$. From a computational modelling framework, prediction error (PE) signals critically rely on DA-dependent signaling in these areas (43), although the prediction error is not limited to reward and punishment processing but also includes sensory-perceptual processes as well as higher order processes such as social learning (43-45).

Against this background we combined a pre-registered randomized double-blind between-group placebo-controlled pharmacological experiment with functional MRI (fMRI) and computational modelling to examine whether social reward and punishment processing can be modulated by a single dose of LT, thus bridging the translational gap between animal model and human research as well as to determine the clinical potential of LT. To this end healthy volunteers $(n=87)$ underwent a wellvalidated social incentive delay (SID) fMRI paradigm (5). Behavioral indices reflecting motivation and subsequent emotional impact of social feedback, neural indices during reward and punishment anticipation and outcome, as well as social feedback PE signalling served as primary outcomes. Based on findings from animal and human studies we hypothesized that LT would (a) enhance differential processing of reward and punishment on the behavioral level (29), which on the neural level would be reflected in (b) enhanced differential activiation and connectivity in VTAstriatal-frontal circuits during social reward-punishment processing, and (c) enhance social feedback PE signalling during the outcome phase.

\section{Materials and method}

\section{Participants}

Ninety healthy participants (age range 18-27 years) were recruited via advertisements for the randomized placebo-controlled between-subject pharmacological fMRI study which encompassed a single-dose p.o. administration of 50mg LT or placebo (PLC) and subsequent administration of a social incentive delay fMRI paradigm (SID) with a demonstrated sensitivity to capture pharmacological modulations (adopted from Nawijn, van Zuiden (5)). Exclusion criteria included color blindness; systolic/diastolic blood pressure $>130 / 90 \mathrm{mmHg}$ or $<90 / 60 \mathrm{mmHg}$; current or regular substance or medication use; current or history of medical or psychiatric disorders; any endocrinological abnormalities or contraindications for LT administration and MRI. 
Participants were asked to abstain from caffeinated drinks on the day of the assessment (e.g., coffee, tea, energy beverages). Two participants were excluded because their baseline blood pressure was outside our predefined criteria, one participant was excluded due to technical failiure during MRI acquisition leading to a final sample of $\mathrm{N}=87(\mathrm{~N}=43,26$ males, $\mathrm{LT} ; \mathrm{N}=44,24$ males, PLC) included in all subsequent analyses (details see Table 1).

\section{Pharmacological and experimental procedure}

Participants were stratified for sex and randomly allocated to treatment in a doubleblind design. Treatment was packed in identical capsules, counterbalanced across sexes and dispensed by an independent researcher. To reduce potential confounding effects of early life stress (46), impulsiveness, sensitivity to punishment and reward on reward-related neural processing the Childhood Trauma Questionnaire (CTQ), Barratt Impulsiveness Scale (BIS), and Sensitivity to Punishment and Sensitivity to Reward Questionnaire (SPSRQ) were administered at baseline (Figure 1A) (47-49). Given that after oral administration LT peak plasma levels are reached after 90 minutes with a terminal elimination half-life ranging from 1.5 to 2.5 hours (50-52) the experimental paradigm started 90 minutes after treatment (in line with $(26,53)$ ). LT rapidly crosses the blood-brain barrier $(54,55)$ and while effects at central receptors have been observed after 30 minutes after i.e. administration effects on cardiovascular indices only become apparent after 3 hours (e.g. $(50)$, see also $(26,29))$. To further control for potential confounding effects of LT on cardiovascular activity blood pressure and heart rate were assessed before drug administration, as well as before and after the fMRI paradigm (Figure 1A). To control for nonspecific affective effects of LT the affective state of participants was tracked troughout the experiment via the Spielberger State-Trait Anxiety Inventory (STAI) and the Positive and Negative Affective Scale (PANAS) which were administered before drug administration, at the time of peak plasma concentrations and after the experiment $(56,57)$ (Figure 1A). The subsequent affective impact of LT-induced changes on social feedback processing was assessed via ratings of the cues before treatment, after fMRI, and following feedback stimuli after fMRI (Figure 1A). After the entire experiment participants were asked to guess the treatment they received.

Written informed consent was obtained from each participant before enrollment, the study was approved by the local institutional ethics committee, all procedure followed the Declaration of Helsinki, and the study was preregistered as clinical trial (registration number: NCT04604756, URL:

https://clinicaltrials.gov/ct2/show/NCT04604756). 
A

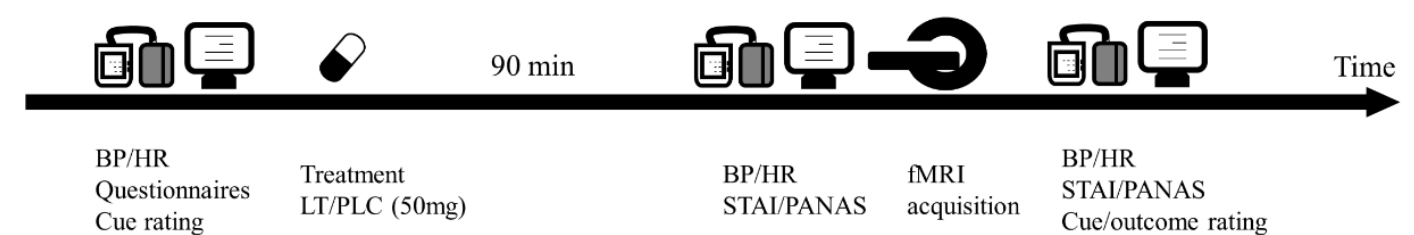

B

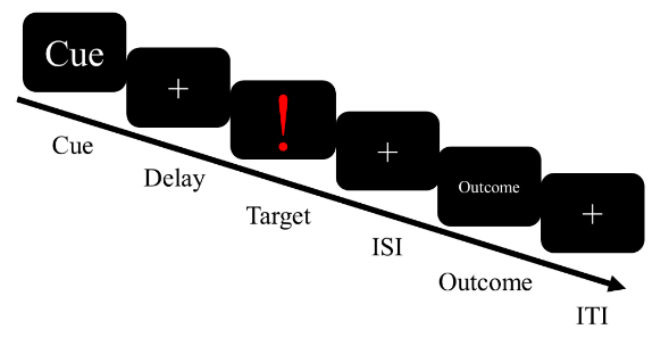

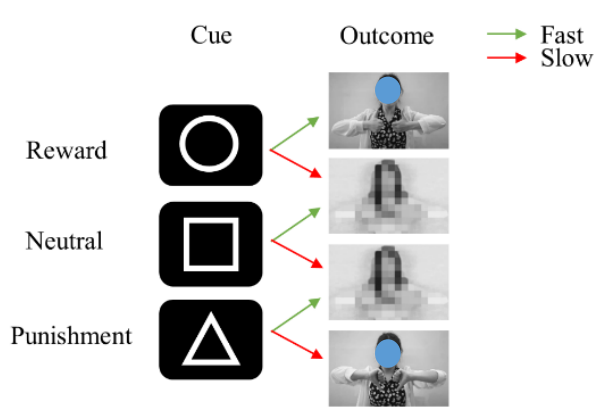

Figure 1. Schematic depiction of the experimental protocols and the experimental paradigm. (A) The entire experimental procedure encompassed baseline assessment, drug administration, assessments before fMRI (corresponding to estimated peak plasma levels) and post fMRI acquisition.

Abbreviations: $\mathrm{BP}=$ blood pressure, $\mathrm{HR}=$ heart rate, $\mathrm{LT}=$ losartan, $\mathrm{PLC}=$ placebo, $\mathrm{STAI}=$ state-trait anxiety inventory, PANAS = positive and negative affect schedule. (B) Schematic representation of the trial structure in the SID paradigm. Each trial started with a 500ms cue presentation (circle, reward trial; square, neutral trials, and triangle, punishment trials) followed by a delay (jittered between 1000 and $3000 \mathrm{~ms}$ ). Next the target appeared with a duration adjusted to the individual response time. After an inter-stimulus interval (ISI, $2000 \mathrm{~ms}$ - target duration) the outcome was presented for $1500 \mathrm{~ms}$, followed by an inter-trial interval (ITI) with a duration jittered between 1000 and $3000 \mathrm{~ms}$. (C) Cues and corresponding outcomes (for display only) per trial type. Social reward feedback was shown in response to fast reactions on reward trails, social punishment feedback was shown in response to slow reactions on punishment trials. Note that for the preprint version the face was overlayed by a blue oval.

\section{Social incentive delay task}

We employed a validated social incentive delay (SID) task-fMRI paradigm with a demonstrated sentivity for pharmacological manipulations (adopted from Nawijn, van Zuiden (5)). Briefly, the paradigm presents condition-specific cues (positive, negative, neutral) which signal that a social reward can be obtained or a social punishment can be avoided (anticipation). Next, participantss undergo a reaction time task which is followed by the presentation of a performance-dependent social reward, punishment or neutral feedback (outcome) (Figure 1B). Participants received task instructions and a practice session prior to the formal experiment. During the paradigm 27 trials for reward and punishment conditions, and 18 trials for the neutral condition were presented (pseudo-randomized). Each trial started with presentation of a geometric cue indicating the trial type (circle: reward, triangle: punishment, and square: neutral, Figure 1C). After a delay, the target was presented in the center of screen and participants were required to press a button as fast as possible. Responses within target presentation time represented hits while omissions or responses outside of 
target presentation time represented misses. To facilitate a sufficient number of trials for each outcome condition an adaptive performance algorithm was employed. To this end, trial-wise reaction times (RT) were recorded and employed to adjust the duration of the next target presentation to individual performance. By increasing or decreasing target durations, $66.7 \%$ of reward-cue and punishment-cue trials were followed by social reward or punishment, respectively. This resulted in a performance-dependent sufficient number of reward (hit) feedback trials $( \pm 18)$ and punishment (miss) feedback trials $( \pm 18)$ for further analyses. The target was followed by an adaptive inter-trial-interval and presentation of the condition- and performance-dependent outcome. In the social reward condition hits resulted in rewarding social feedback, i.e. a smiling person in thumbs-up pose, while misses resulted in neutral feedback, i.e. scrambled picture of the person. In the social punishment condition hits allowed avoidance of social punishment (neutral feedback), while misses resulted in social punishment feedback, i.e. a person with a contemptuous look in thumbs-down pose. For neutral trials both, hits and misses resulted in neutral feedback. The experimental materials for the outcome were initially collected and rated by an independent sample. To further explore the subsequent emotional effects of the paradigm participants rated perceived arousal, likeability, dislikeability, intensity, valence of cues and outcomes on a 9-point Likert scale after the fMRI session (Figure 1A).

\section{Behavioral analysis}

To maintain the trial-specific information of the SID task and increase sensitivity, a linear mixed model was used with condition (social reward, punishment, neutral) and treatment (LT, PLC) as two fixed factors and subject as random factor to account for individual adaptations of reaction time windows. Trials with no responses and RTs $\pm 3 \mathrm{SD}$ on the individual level were removed. Treatment effects on emotional perception ratings of cues and outcomes were examined with separate ANOVA and liner mixed models (see supplementary method).

\section{MRI data acquisition and preprocessing}

MRI data was acquired using a 3.0 Tesla GE MR750 system (General Electric Medical System, Milwaukee, WI, USA). Preprocessing was fully implemented in fMRIPrep (58) except for smoothing with a Gaussian kernel at full width at half maximum (FWHM, $8 \times 8 \times 8 \mathrm{~mm}$ ) conducted in SPM12 (Welcome Department of Cognitive Neurology, London, UK, http:/www.fil.ion.ac.uk/spm/software/spm12) (59). Details please see supplementary method.

\section{Individual- and group-level BOLD level fMRI analyses}

On the first-level the SID task was modeled employing the hemodynamic response function (HRF) and corresponding derivatives on the onsets of the experimental conditions, i.e. cue, target, and outcome. The three different types of anticipation were modeled according to the cues signaling a potential social reward, social punishment, and neutral outcome. In line with previous studies the cue and subsequent delay were modelled as anticipation period $(2,60-62)$. In line with the potential response pattern 
of the participants five different types of outcome were modeled, including reward or neutral feedback in reward trial, punishment or neutral feedback in punishment trial, and neutral feedback in neutral trial. Additional confound regressors included target responses and head motion as defined by six rigid movement parameters from the motion correction. On the group level effects of treatment were examined employing mixed ANOVA analyses with the factors (condition, treatment) for each phase. Based on our a priori regional hypotheses the analyses focused on the ventral striatum (VS), dorsal striatum (DS) (63) and VTA (64) as Regions of Interest (ROIs).

\section{Functional Connectivity Analysis}

To determine the social reward-punishment networks we initially examined neural activity during receipt of feedback [reward + punishment - neutral] in the entire sample. Results revealed that social feedback induced stronger activity in regions involved in salience, value and social processes, including insula, striatum, dorsal medial prefrontal cortex (dmPFC), and occipital lobe (Figure S1C). Combined with the a priori defined VTA-striatal structural maks three peak coordinates (VS: [22, -6, 10], DS: $[-14,-2,-8]$, VTA: $[10,-14,-12])$ were identified to construct spherical seeds with $6 \mathrm{~mm}$ radius which served as seeds for the generalized psychophysiological interactions (gPPI) analysis (65) (supplementary method). Treatment effects were determined by comparing the seed-region-specific connectivity maps by means of mixed ANOVA analyses with the factors (condition, treatment) on the whole brain level (separately for each phase). To further disentangle significant interaction effects parameter estimates were extracted from regions exhibiting significant interaction effects involving treatment.

\section{Computational modelling of social feedback prediction errors on the neural level}

Due to the consistent information carried by cue and outcome in neutral condition, the prediction error for each trial was only computed for the social reward and social punishment conditions and employed as a trial-wise parametric modulator on the neural level (66). The prediction error (PE) and expected value (EV) for each trial were estimated based on the Rescorla-Wagner model (66-68). The value estimation was based on the equations:

$$
\begin{gathered}
P E_{t}=R_{t}-E V_{t} \\
E V_{t+1}=E V_{t}+\alpha \times P E_{t}
\end{gathered}
$$

$P E$ reflects the difference between the expectation and the actual outcome, and EV reflects the expectation of receiving a certain feedback on a given trail. $\mathrm{R}$ is the actual feedback received, $\mathrm{t}$ is the given trial, and $\alpha$ is the learning rate. The initial EV for social reward anticipation and social punishment anticipation were set up with 0.5 and -0.5 respectively. In line with previous studies $(66,69)$ a learning rate $\alpha=0.7$ was used across participants. The EV for the next trial is updated based on the EV of the current trial and the prediction error of that trial multiplied by the learning rate. Since previous studies show that a wide range of learning rates ( 0.2 to 0.7$)$ do not affect the computation $(66,67,69-71)$, an additional test was conducted by calculating at a 
learning rate of 0.2 , which confirmed that changing the learning rate did not affect our main findings (see supplementary results).

For determining the social feedback PE on the neural level EV and PE were included in the first level GLM models as parameter modulations corresponding to anticipation and outcome phase respectively. On the first level the GLM models corresponded to the BOLD activation models and the the expectation value and prediction error from the computational model were added into the first-level model as additional parameter modulations for the anticipation and outcome respectively. Based on previous studies $(66,69)$ outcome stage was modeled without taking specific feedback into account to avoid overfitting. Treatment effects on the second level were examined by means of directly comparing the two treatment groups by means of independent $t$ tests.

\section{Thresholding}

ROI analyses were conducted in the R package 'afex', and the statistical significance level set to $p<.05$. On the whole-brain level an tinitial cluster-forming threshold was set to voxel level $p<.001$, and statistical significance was determined via clusterlevel inference and familywise error (FWE) control for multiple comparisons with $p_{F W E}<.05$ (72).

\section{Results}

\section{Participants}

Treatmemnt groups (losartan, $\mathrm{n}=43$; placebo, $\mathrm{n}=44$ ) exhibited comparable sociodemographic and psychometric characteristics (Table 1). During the experiment no differences in baseline assessments or changes in heart rate, blood pressure, and emotional state were observed between the treatment groups and total guess accuracy was $52.87 \%$ together arguing against the impact of potential confounders and unspecific effects of LT.

Table 1. Participant Demographics and Control Measures

\begin{tabular}{|c|c|c|c|c|c|}
\hline Characteristic & Time & LT, $\mathrm{N}=43$ & PLC, $N=44$ & Statistic & $p$ value \\
\hline Age, years & & $21.56(2.29)$ & $20.84(1.94)$ & 1.6 & 0.119 \\
\hline Sex & & & & 0.12 & 0.733 \\
\hline Male & & $26(60 \%)$ & $24(55 \%)$ & & \\
\hline Female & & $17(40 \%)$ & $20(45 \%)$ & & \\
\hline Body mass index, $\mathrm{kg} / \mathrm{m}^{2}$ & & $21.02(2.38)$ & $21.33(3.35)$ & -0.49 & 0.622 \\
\hline CTQ & & $36.81(7.89)$ & $34.59(5.36)$ & 1.5 & 0.129 \\
\hline BIS- AI & & $14.35(2.91)$ & $14.18(2.31)$ & 0.30 & 0.768 \\
\hline BIS- MI & & $22.84(3.73)$ & $22.11(3.95)$ & 0.88 & 0.382 \\
\hline BIS- NPI & & $23.65(4.23)$ & $23.55(4.09)$ & 0.12 & 0.906 \\
\hline
\end{tabular}




\begin{tabular}{|c|c|c|c|c|c|}
\hline SPSR- SP & & $24.40(4.58)$ & $25.20(3.52)$ & -0.92 & 0.359 \\
\hline SPSR-SR & & $23.02(2.51)$ & $22.48(3.37)$ & 0.86 & 0.393 \\
\hline \multirow[t]{3}{*}{ Systolic blood pressure } & Baseline & $116.42(7.60)$ & $114.55(9.01)$ & 1.0 & 0.297 \\
\hline & Before MRI & $110.26(8.94)$ & $110.18(9.64)$ & 0.04 & 0.970 \\
\hline & After MRI & $115.40(8.12)$ & $112.98(9.80)$ & 1.3 & 0.213 \\
\hline \multirow[t]{3}{*}{ Diastolic blood pressure } & Baseline & $72.42(6.59)$ & $70.89(6.93)$ & 1.1 & 0.294 \\
\hline & Before MRI & $69.16(6.83)$ & $67.61(6.21)$ & 1.1 & 0.272 \\
\hline & After MRI & $71.67(6.38)$ & $70.18(8.12)$ & 1.0 & 0.342 \\
\hline \multirow[t]{3}{*}{ Heart rate } & Baseline & $80.05(12.50)$ & $76.05(11.31)$ & 1.6 & 0.121 \\
\hline & Before MRI & $70.84(11.23)$ & $69.73(8.36)$ & 0.52 & 0.603 \\
\hline & After MRI & $69.70(11.29)$ & $71.52(11.44)$ & -0.75 & 0.456 \\
\hline \multirow[t]{3}{*}{ PANAS- negative affect } & Baseline & $16.58(4.99)$ & $15.57(4.41)$ & 1.0 & 0.319 \\
\hline & Before MRI & $14.68(4.67)$ & $13.73(3.82)$ & 1.0 & 0.323 \\
\hline & After MRI & $13.82(4.37)$ & $13.74(4.89)$ & 0.08 & 0.932 \\
\hline \multirow[t]{3}{*}{ PANAS- positive affect } & Baseline & $27.16(5.97)$ & $26.77(5.51)$ & 0.32 & 0.752 \\
\hline & Before MRI & $25.88(6.38)$ & $24.93(5.55)$ & 0.71 & 0.478 \\
\hline & After MRI & $24.68(7.02)$ & $24.67(5.93)$ & 0.01 & 0.995 \\
\hline \multirow[t]{3}{*}{ STAI- state anxiety } & Baseline & $38.98(7.27)$ & $39.41(6.84)$ & -0.29 & 0.776 \\
\hline & Before MRI & $38.50(7.57)$ & 38.78 (7.87) & -0.16 & 0.870 \\
\hline & After MRI & $38.92(8.67)$ & $38.86(8.05)$ & 0.04 & 0.971 \\
\hline \multirow[t]{3}{*}{ STAI- trait anxiety } & Baseline & $41.35(8.16)$ & $40.82(7.71)$ & 0.31 & 0.756 \\
\hline & Before MRI & $40.65(7.74)$ & $40.54(7.75)$ & 0.07 & 0.948 \\
\hline & After MRI & $40.58(8.32)$ & $41.00(8.23)$ & -0.23 & 0.817 \\
\hline
\end{tabular}

${ }^{a}$ Descriptive statistics: mean (SD); n (\%)

b Statistical tests: Welch Two Sample t-test; Pearson's Chi-squared test

${ }^{\mathrm{c}} \mathrm{LT}=$ losartan, $\mathrm{PLC}=$ placebo, PANAS $=$ Positive and Negative Affect Schedule, $\mathrm{STAI}=$ Spielberger State-Trait Anxiety Inventory, CTQ = Childhood Trauma Questionnaire, BIS = Barratt Impulsiveness Scale (AI = attentional impulsiveness, $\mathrm{MI}=$ motor impulsiveness, NPI = non-planning impulsiveness $)$, SPSR $=$ Sensitivity to Punishment and Sensitivity to Reward questionnaire ( $\mathrm{SP}=$ sensitivity to punishment, $\mathrm{SR}=$ sensitivity to reward)

${ }^{\mathrm{d}}$ Due to technological issues of PANAS and STAI assessments 3 recordings in LT group and 2 recordings in PLC group were lost during before and after MRI phases.

\section{Losartan and experimental effects on motivational significance and affective evaluation}

The linear mixed model revealed a significant interaction effect $(F=3.706, p=0.025$, Figure 2A) between condition and treatment on reaction times indicating that LT induced significantly stronger differences between social punishment vs social reward as compared to PLC $(t=2.679, p=0.007)$, reflecting a shift in the approach- 
avoidance motivation of social feedback. The main effects of treatment and condition were not significant.

Examining effects of the experimental manipulation and treatment on the affective evaluation by means of a linear mixed model revealed a significant condition main $(F=404.983, p<0.0001$, Figure 2B) and condition times treatment interaction effect $(F=4.914, p=0.007)$ on arousal ratings for outcomes. Post hoc analyses showed that LT increased the reward-punishment difference $(t=2.390, p=0.017)$ and decreased punishment-neutral difference $(t=-2.952, p=0.003)$ relative to PLC. With respect to dislikeability ratings for the outcomes a linear mixed model revealed significant condition main $(F=633.848, p<0.0001$, Figure 2C), and condition times treatment interaction effects $(F=3.413, p=0.033)$. Post hoc tests showed that LT increased the punishment-neutral difference $(t=2.597, p=0.0095)$ relative to PLC. No significant treatment main or interaction effects were observed on cue or other outcome ratings (see supplementary results).

A

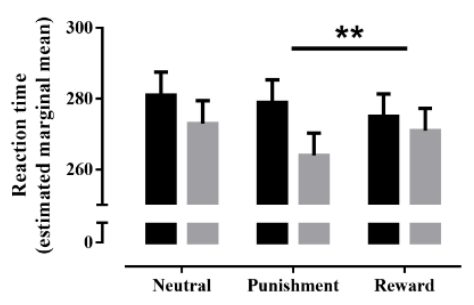

B

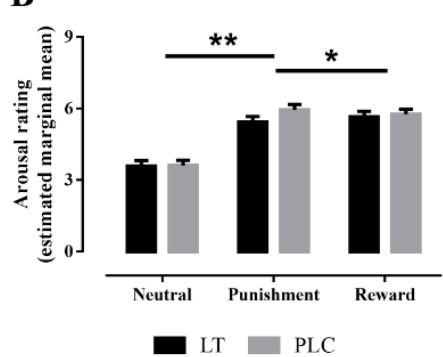

C

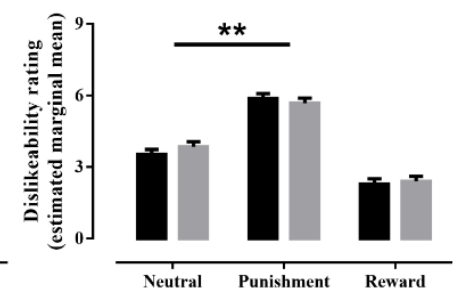

Figure 2. Reaction time, arousal and dislikeability ratings of the feedback pictures presented during outcome. Estimated marginal mean and stand error of reaction time (A), arousal rating (B), and dislikeability rating $(\mathrm{C})$ are presented. * and $* *$ denote relevant significant post hoc differences at $p<$ 0.05 and $p<0.01$ respectively. $\mathrm{LT}=$ losartan, PLC $=$ placebo.

\section{Losartan effects on neural activation during anticipation phase}

No significant main or interaction effects of treatment were observed in the a priori ROI analyses on extracted parameter estimated from the VTA (treatment, $F=2.67, p$ $=0.106$; interaction, $F=0.309, p=0.724$ ), VS (treatment, $F=1.277, p=0.2616$; interaction, $F=0.218, p=0.7802$ ), and DS (treatment, $F=0.96, p=0.33$; interaction, $F=0.65, p=0.5165$ ). In addition an exploratory whole-brain analysis confirmed the lack of significant treatment main and interaction effects (at $p_{F W E}<0.05$ ). Significant main effects of condition during the anticipation phase are provided in

\section{supplementary results (Figure S1A, Table S1).}

\section{Losartan effects on neural activation during outcome phase}

The ROI analysis revealed a significant treatment times condition effect on the VTA $(F=3.24, p=0.0435)$, reflecting that LT significantly increased the difference between reward and neutral $(t=2.407, p=0.0172)$ as well as between reward and punishment ( $t=1.924, p=0.056$, marginal significant). The exploratory whole-brain analysis did not reveal further regions exhibiting treatment effects suggesting highly 
specific regional effects of LT. Significant main effects of condition during the anticipation phase are provided in supplementary results (Figure S1B, Table S1).

\section{Losartan effects on the network level}

On the network level significant interaction effects between condition and treatment were found for the VS during anticipation but for the VTA during the outcome phase (all findings passed whole-brain $p_{F W E}<.05$, Figure 3, Table 2). Subsequent post-hoc tests revealed that LT significantly modulated VS-middle frontal gyrus (MFG) connectivity during neutral-punishment $(t=2.541, p=0.0119)$, punishment-reward $(t$ $=-3.910, p=0.0001)$, and between social reward feedback $(t=2.451, p=0.0151)$ processes, with the effects being driven by enhanced coupling during social rewardanticipation. In contrast, LT specifically modulated VTA-networks during outcome, such that LT modulated VTA-insula (left) connectivity during the neutral-punishment pattern $(t=2.613, p=0.0098)$, the punishment-reward pattern $(t=-4.671, p<$ $0.0001)$, the neutral-reward pattern $(t=-2.059, p=0.0410)$, and within social punishment $(t=-2.012, p=0.0456)$ and social reward $(t=3.128, p=0.002)$ respectively. In addition, LT modulated both VTA-insula (right) and VTA-SFG connectivity for neutral-punishment $(t=5.023, p<0.0001 ; t=-3.127, p=0.0021)$ and punishment-reward patterns $(t=-3.683, p=0.0003 ; t=4.368, p<0.0001$, Figure 3C). Losartan also changed VTA-insula (right) connectivity in social punishment $(t=$ -2.512, $p=0.0128)$ and neutral $(t=3.13, p=0.002)$, VTA- superior frontal gyrus (SFG) connectivity in social punishment $(t=3.613, p=0.0004)$. A direct comparion between treatments revealed consistent effects of LT on processing of social punishment feedback, such that it decreased VTA communication with the bilateral insula, yet enhanced VTA communication with the SFG. 


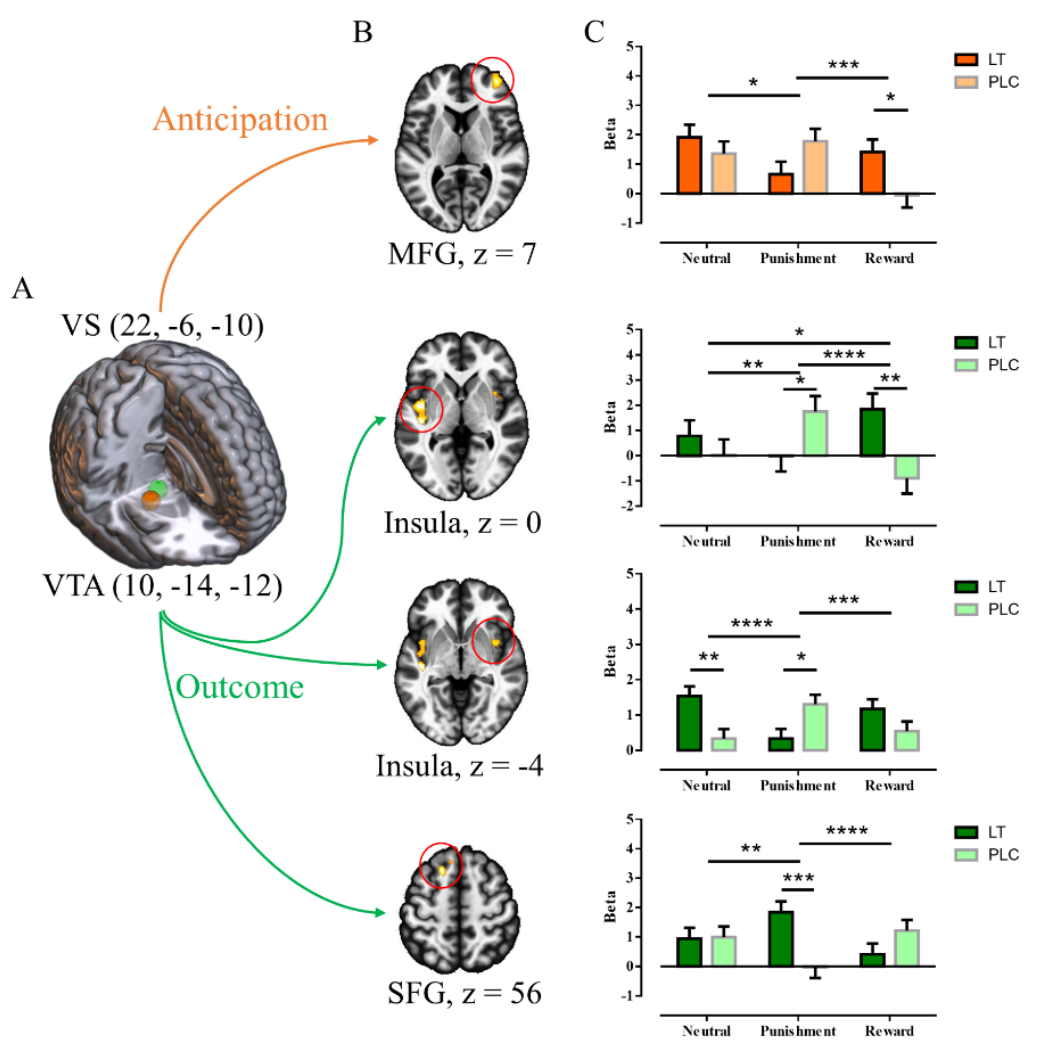

Figure 3. Effects of LT on the network level. (A) Seeds of interest, i.e. VS and VTA. (B) Regions exhibiting significant conditions times treatment interaction effects during anticipation and outcome phases. (C) Post hoc tests on extracted parameters from each significant clusters. VS = ventral striatum $\mathrm{VTA}=$ ventral tegmental area, $\mathrm{SFG}=$ superior frontal gyrus, $\mathrm{MFG}=$ middle frontal gyrus, $\mathrm{LT}=$ losartan, PLC $=$ placebo, $* * *, * * *$, and $* * * *$ denote relevant significant post hoc differences at $p<$ $0.05, p<0.01, p<0.001$, and $p<0.0001$ respectively.

Table 2 Functional connectivity results

\begin{tabular}{cccccc}
\hline Cluster region & Cluster size & $\mathrm{x}$ & $\mathrm{y}$ & $\mathrm{z}$ & $F$ value \\
\hline Anticipation phase, VS seed & & & & & \\
MFG & 309 & 38 & 56 & 8 & 13.69 \\
Outcome phase, VTA seed & & 38 & 60 & 16 & 11.41 \\
& & & & & \\
L insula, STG & 427 & -44 & -22 & -2 & 15.16 \\
& & -44 & -6 & 0 & 12.50 \\
R insula, putamen & 246 & -40 & 6 & -6 & 10.44 \\
& & 40 & 12 & 16 & 14.09 \\
SFG & & 38 & 4 & -6 & 11.07 \\
& 213 & -18 & 20 & 56 & 11.75 \\
& & -8 & 30 & 60 & 9.83 \\
& & -30 & 22 & 46 & 8.26 \\
\hline
\end{tabular}

Note: All clusters passed the threshold at whole-brain cluster level $p_{F W E}<.05 . \mathrm{L}=$ left, $\mathrm{R}=$ right, $\mathrm{SFG}$ $=$ superior frontal gyrus, $\mathrm{MFG}=$ middle frontal gyrus, $\mathrm{STG}=$ superior temporal gyrus. 


\section{Losartan effects on the social feedback prediction error}

The ROI analysis indicated that LT significantly enhanced the social feedback PE in $\operatorname{DS}(t=2.454, p=0.0162)$ and VTA $(t=2.227, p=0.0286)$ but not the VS $(t=1.264$, $p=0.2098$ ) compared to PLC (Figure 4). Further exploratory whole brain analysis revealed both significant positive and negative social feedback PE modulation in widespread subcortical-cortical networks (Figure 5A \& 5B, Table 3), while LT enhanced the social feedback PE in a cortical midline and superior temporal network encompassing the precuneus, STG, SFG, MFG, cingulate gyrus and SMA (Figure 5C, Table 3).

A

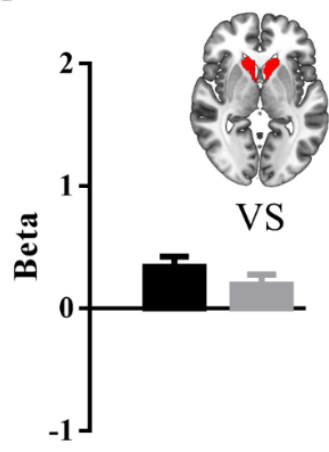

B

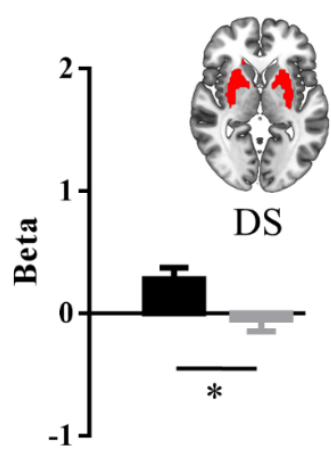

$\mathrm{C}$

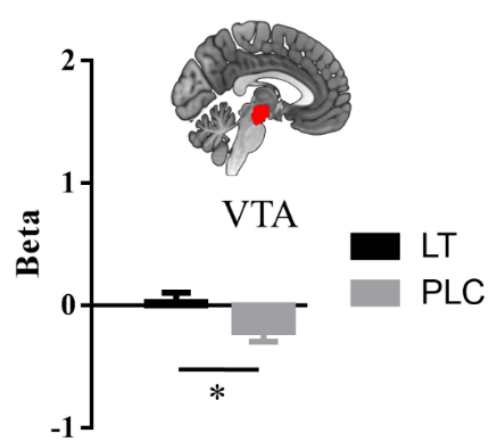

Figure 4. Parameter estimates reflecting extracted neural PE signals from the corresponding midbrain and striatal regions. $*$ denote relevant significant post hoc differences at $p<0.05 . \mathrm{VS}=$ ventral striatum, DS $=$ dorsal striatum, VTA $=$ ventral tegament area, $\mathrm{LT}=$ losartan, $\mathrm{PLC}=$ placebo. 
A. Positive effects of PE

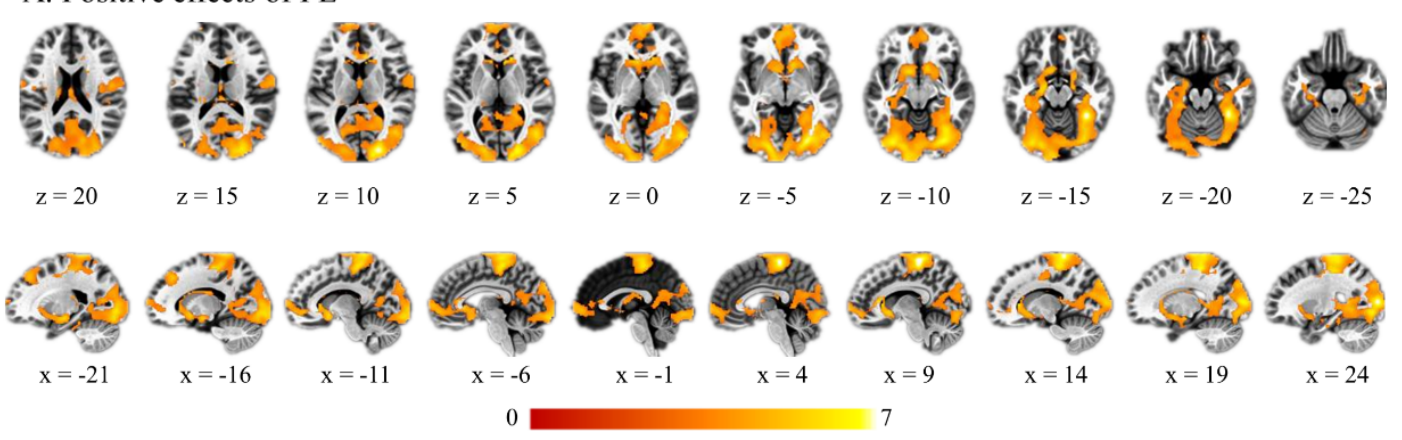

B. Negative effects of PE
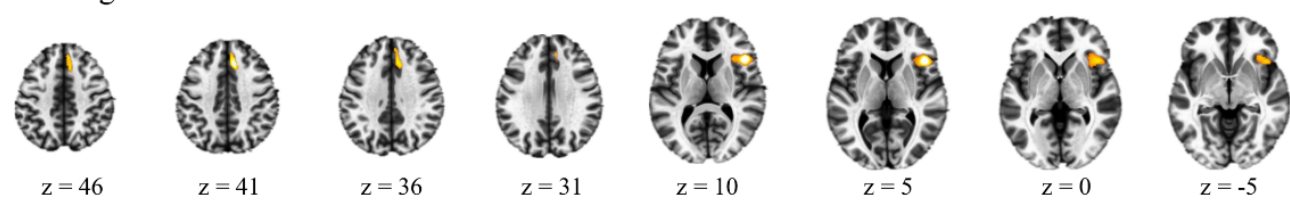

\section{LT $>$ PLC}
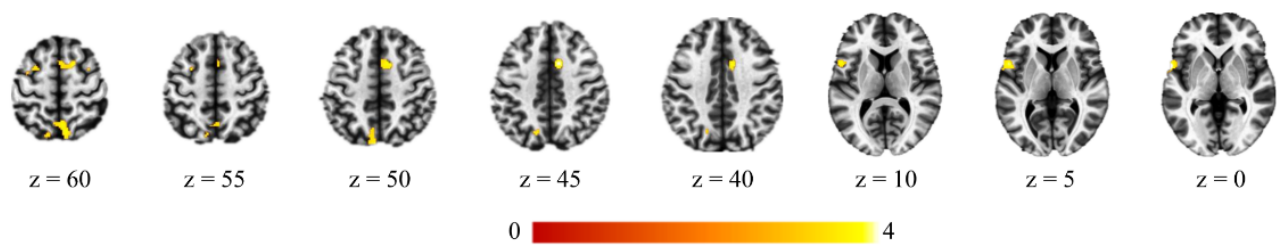

Figure 5. Prediction error (PE) related neural signals. (A) Positive effects of PE, (B) negative effects of $\mathrm{PE}$, and (C) regions exhibiting an enhanced PE by losartan compared to placebo. All clusters passed the threshold at whole-brain cluster level $p_{F W E}<.05 . \mathrm{LT}=$ losartan, PLC $=$ placebo.

Table 3 Neural correlates of prediction error

\begin{tabular}{cccccc}
\hline Cluster region & Cluster size & $\mathrm{x}$ & $\mathrm{y}$ & $\mathrm{z}$ & $t$ value \\
\hline Positive effects of PE & & & & & \\
Parietal Lobe, mPFC, SFG, & 12731 & 8 & -30 & 66 & 8.13 \\
Precuneus & & 14 & -38 & 74 & 7.22 \\
& & 20 & -34 & 56 & 6.83 \\
Occipital Lobe, Temporal & 28095 & 36 & -48 & -16 & 7.92 \\
Lobe, subcortical regions* & & 24 & -92 & 8 & 7.21 \\
& & 32 & -80 & -8 & 7.12 \\
vmPFC, SFG, MFG, mPFC & 733 & -18 & 34 & 44 & 5.36 \\
Negative effects of PE & & -24 & 20 & 32 & 3.84 \\
IFG, insula & 707 & 46 & 20 & 8 & 6.47 \\
& & 36 & 26 & -4 & 4.37 \\
mPFC, cingulate gyrus & & 32 & 22 & 12 & 4.05 \\
LT $>$ PLC & 417 & 8 & 28 & 40 & 5.42 \\
& & 10 & 38 & 26 & 3.87 \\
\hline
\end{tabular}




\begin{tabular}{cccccc}
\hline SFG, MFG, cingulate gyrus, & 1891 & 12 & -4 & 76 & 5.09 \\
SMA & & -18 & -16 & 78 & 4.94 \\
& & 28 & -4 & 68 & 4.19 \\
STG & 316 & -60 & 6 & 0 & 4.25 \\
& & -54 & 10 & 8 & 4.08 \\
Precuneus & \multirow{2}{*}{728} & -56 & 6 & 18 & 3.24 \\
& & 10 & -66 & 64 & 4.05 \\
& & -16 & -70 & 44 & 3.79 \\
\hline
\end{tabular}

Note: All clusters passed the threshold at whole-brain cluster level $p_{F W E}<.05 . \mathrm{LT}=$ losartan, PLC $=$ placebo, $\mathrm{IFG}=$ inferior frontal gyrus, $\mathrm{SFG}=$ superior frontal gyrus, $\mathrm{mPFC}=$ medial prefrontal cortex, $\mathrm{vmPFC}=$ ventral medial prefrontal cortex, $\mathrm{MFG}=$ middle frontal gyrus, $\mathrm{STG}=$ superior temporal gyrus, $\mathrm{MTG}=$ middle temporal gyrus, $\mathrm{SMA}=$ supplementary motor area. * parahippocampal gyrus, caudate, putamen, amygdala, thalamus.

\section{Exploratory analyses of sex-differences}

Potential sexual dimorphic effects of LT were examined in exploratory analyses encompassing the factor sex. No sex-differences were observed, except for a stronger LT effect on the striatal PE in men (supplementary results).

\section{Discussion}

The present pharmacological fMRI trial aimed to determine whether targeting the RAS system via LT can modulate social reward and punishment processing via modulating VTA-striatal-frontal circuits. On the behavioral level LT modulated the motivational significance of social reward and punishment during anticipation while affecting the subsequent affective evaluation of social stimuli. On the neural level the enhanced motivational significance was reflected by increased coupling between the VS and MFG during anticipation of social rewards. During the outcome phase LT enhanced neural signals in the VTA and associated prediction error signals in VTA and DS while attenuating VTA-insula communication and concomitantly enhancing VTA-SFG communication during social punishment. Together, these findings provide a potential mechanism by which LT may enhance social reward motivation while decreasing social punishment sensitivity.

On the behavioral level LT shifted the motivational significance and arousal experience for social punishment relative to social reward feedback, an effect that was mainly driven by prolonged reaction times during anticipation of and subsequently reduced arousal reaction towards social punishment stimuli. These findings partly align with observations in previous studies, such that following LT healthy subjects perceived loss outcomes as being less informative resulting in an attenuated loss learning rate (29), and exhibited accelerated extinction and autonomous arousal decreases towards threat (26). Together, these observations indicate that LT may attenuate the impact of negative information thus shifting anticipatory motivation and post encounter learning towards positive information. 
On the neural level the relative motivational shift between negative and positive social information was accompanied by a modulation of VS-frontal circuits, such that LT reduced VS-MFG connectivity during anticipation of social punishment but increased connectivity in this circuit during anticipation of social reward. Convergent evidence suggests that the VS plays a key role in dopamine-mediated anticipatory and motivational processes $(17,18,42)$ and that the pathways between the VS and frontal regions are critically involved in associated social processes including motivational and reinforcing aspects of social interactions $(73,74)$. In patients with marked social impairments pharmacological modulation of the coupling between VS and MFG has been associated with improved computation of future positive social outcomes ( 75 , 76) and effects on this circuit may thus reflect a potential mechanism via which LT can increase social motivation.

In contrast to the modulation of VS-centered circuits during the anticipation stage, LT specifically modulated VTA activity as well as its connectivity with insular and frontal regions during the outcome phase. During the social feedback presentation stage LT increased the differential processing of rewarding feedback from both, negative as well as neutral feedback in the VTA. The VTA represents a pivotal node in dopamine-modulated reward processing and learning circuits $(17,22,40,77)$ and together with the amygdala drives dopaminergic signaling in response to social stimuli $(22,74)$, suggesting that LT rendered positive social signals as more salient. In contrast, LT specifically decreased coupling of the VTA with the bilateral midposterior insula in response to social punishment. The insula plays a key role in salience and interoceptive information processing, with the mid-posterior insula being involved in representing the intensity of aversive experiences $(78,79)$. This suggests that LT may have attenuated the aversive emotional impact of negative social feedback on the insula leading to lower arousal ratings for the negative social stimuli following the experiment.

We further combined a computational modeling framework to determine effects of LT on the neural social prediction error i.e. the difference between expected and actual social feedback. The feedback evoked PE signal is a closely linked to the dopamine transmission based neural teaching signal and thus promotes behavioral adaptations to the external environment $(38,39,44)$. LT enhanced the social feedback PE signal on the neural level in the VTA and DS and convergent evidence suggest that the PE signal is strongly influenced by dopaminergic signalling. Previous animal models suggest interactions between the RAS and the DA system, such that LT activiated D1R $(34)$ and inhibited DA release $(32,33)$ in these circuits, which may suggest that LT enhanced PE encoding in these regions via effects on DA neurons rather than on postsynaptic dopaminergic transmission. In addition, LT also enhanced PE signaling in other regions, including frontal and superior temporal areas as well as the precuneus. Whereas RPE signals have been mostly examined in midbrain and striatal regions, accumulating evidence suggests that depending on the specific domains and context more extended networks encompassing limbic, frontal and parietal regions are involved $(43,44)$. For instance, the concommitant engagement of the $\mathrm{mPFC}$, amygdala, hippocampus with the precuneus and the default network has 
been proposed to play a key role in initiating and maintaining exploratory and exploitative behavior $(45,77,80)$, while the insula has been strongly associated with negative PEs signaling sensitivity to punishment $(24,81,82)$. The regions affected by LT encompass onesinvolved in executive functions and social processes such as self referential processing and theory of mind (80) suggesting that LT in the present context may have modulated not only reward-related PE signals but also other components of the PE.

From a functional neuroanatomy perspective LT modulated neural activity and connectivity of distinct key nodes of the midbrain-striatal system during different aspects of social feedback processing. Thus, VS connectivity was specifically affected during anticipation while VTA networks and VTA/DS PE signaling were modulated during the outcome phase. This dissociation aligns with the distinct functions of these core nodes in feedback-associated social and non-social processes $(63,83,84)$. The VTA encompasses the majority of dopaminergic cell bodies and is strongly involved in predicting outcomes including social error signals and guiding flexible adaptation $(17,22,46,85)$, whereas the VS which receives dopaminergic projections from the VTA, is strongly involved in appetitive motivation and rewardexpectation for both social and non-social feedback $(17,18)$ while the DS is stronger involved in learning, action initiation, and habit formation $(16,83)$. Altough most of these functions encompass social as well as non-social processes their critical role in reward and punishment processing critically influences social behavior $(45,73,74)$. The process-specific effects of LT on distinct nodes may reflect that the RAS plays a complex role in regulating social reward and punishment processes.

Social deficits such as decreased social motivation or a hypersensitivity to social punishment represent a core symptom across several mental disoders including depression $(2,3)$, social anxiety disorder (4), post-traumatic stress disorder $(5,15)$, autism spectrum disorder $(7,8)$, and schizophrenia (9). Together with accumulating evidence from previous studies $(26,27,29)$ our findings suggest that LT may have a promising potential to enhance social motivation to obtain rewards while decreasing sensitivity to punishment in social contexts and attenuate these dysregulations in patient populations.

Although the current study employed a strict pre-registered and randomizedcontrolled design the findings and interpretation need to be considered in the context of the following limitations. First, due to the proof-of-concept design the study was conducted in healthy individuals. Although this allowed us to control for a range of potential confounders, effects in patients and on the symptomatic level need to be systematically examined (27). Second, although the findings suggest that interactions between the RAS and the DA system may have contributed to the observed effect no direct measures of DA functioning were assessed and future molecular imaging studies are need.

In conclusion the present findings demonstrate that targeting the RAS via LT modulates the VTA-striatal-frontal cicruits during social reward and punishment processing. LT shifted the motivational significance of social reward vs punishment feedback and concomitantly modulated the VS-prefrontal pathways. During the 
outcome phase LT attenuated VTA-insula coupling during social punishment yet enhanced the social PE in the VTA and DS suggesting attenuated sensitivity to social punishment in the context of enhanced adaptation. Together with the excellent safety profile of LT the findings may suggest a therapeutic property to enhance social motivation and attenuate the impact of negative social feedback. 


\section{Acknowledgments and disclosures}

This study was supported by the National Key Research and Development Program of China (2018YFA0701400). Data availiability: unthresholded group-level statistical maps are available via the OSF (https://osf.io/mnda4/) other data of this study is available from the corresponding author upon reasonable request. The authors report no biomedical financial interests or potential conflicts of interest. 


\section{References}

1. Frey AL, Frank MJ, McCabe C (2021): Social reinforcement learning as a predictor of real-life experiences in individuals with high and low depressive symptomatology. Psychol Med. 51:408-415.

2. Zhang D, Shen J, Bi R, Zhang Y, Zhou F, Feng C, et al. (2020): Differentiating the abnormalities of social and monetary reward processing associated with depressive symptoms. Psychol Med.1-15.

3. Russo SJ, Nestler EJ (2013): The brain reward circuitry in mood disorders. Nat Rev Neurosci. 14:609-625.

4. Cremers HR, Veer IM, Spinhoven P, Rombouts SA, Roelofs K (2014): Neural sensitivity to social reward and punishment anticipation in social anxiety disorder. Front Behav Neurosci. 8:439.

5. Nawijn L, van Zuiden M, Koch SB, Frijling JL, Veltman DJ, Olff M (2017): Intranasal oxytocin increases neural responses to social reward in post-traumatic stress disorder. Soc Cogn Affect Neurosci. 12:212-223.

6. Tobler PN, Preller KH, Campbell-Meiklejohn DK, Kirschner M, Kraehenmann R, Stampfli P, et al. (2016): Shared neural basis of social and non-social reward deficits in chronic cocaine users. Soc Cogn Affect Neurosci. 11:1017-1025.

7. Delmonte S, Gallagher L, O'Hanlon E, McGrath J, Balsters JH (2013): Functional and structural connectivity of frontostriatal circuitry in Autism Spectrum Disorder.

Front Hum Neurosci. 7:430.

8. Kinard JL, Mosner MG, Greene RK, Addicott M, Bizzell J, Petty C, et al. (2020): Neural Mechanisms of Social and Nonsocial Reward Prediction Errors in Adolescents with Autism Spectrum Disorder. Autism Res. 13:715-728.

9. Mow JL, Gandhi A, Fulford D (2020): Imaging the "social brain" in schizophrenia: A systematic review of neuroimaging studies of social reward and punishment. Neurosci Biobehav Rev. 118:704-722.

10. Kendrick KM, Guastella AJ, Becker B (2018): Overview of Human Oxytocin Research. Curr Top Behav Neurosci. 35:321-348.

11. Quintana DS, Lischke A, Grace S, Scheele D, Ma Y, Becker B (2021): Advances in the field of intranasal oxytocin research: lessons learned and future directions for clinical research. Mol Psychiatry. 26:80-91.

12. Cuthbert BN, Insel TR (2013): Toward the future of psychiatric diagnosis: the seven pillars of RDoC. BMC Med. 11:126.

13. Der-Avakian A, Markou A (2012): The neurobiology of anhedonia and other reward-related deficits. Trends Neurosci. 35:68-77.

14. Luijten M, Schellekens AF, Kuhn S, Machielse MW, Sescousse G (2017):

Disruption of Reward Processing in Addiction : An Image-Based Meta-analysis of Functional Magnetic Resonance Imaging Studies. JAMA Psychiatry. 74:387-398.

15. Fenster RJ, Lebois LAM, Ressler KJ, Suh J (2018): Brain circuit dysfunction in post-traumatic stress disorder: from mouse to man. Nat Rev Neurosci. 19:535-551. 16. Klugah-Brown B, Di X, Zweerings J, Mathiak K, Becker B, Biswal B (2020): Common and separable neural alterations in substance use disorders: A coordinate- 
based meta-analyses of functional neuroimaging studies in humans. Hum Brain Mapp. 41:4459-4477.

17. Gu R, Huang W, Camilleri J, Xu P, Wei P, Eickhoff SB, et al. (2019): Love is analogous to money in human brain: Coordinate-based and functional connectivity meta-analyses of social and monetary reward anticipation. Neurosci Biobehav Rev. 100:108-128.

18. Martins D, Rademacher L, Gabay AS, Taylor R, Richey JA, Smith DV, et al. (2021): Mapping social reward and punishment processing in the human brain: A voxel-based meta-analysis of neuroimaging findings using the social incentive delay task. Neurosci Biobehav Rev. 122:1-17.

19. Dolen G, Darvishzadeh A, Huang KW, Malenka RC (2013): Social reward requires coordinated activity of nucleus accumbens oxytocin and serotonin. Nature. 501:179-184.

20. Hung LW, Neuner S, Polepalli JS, Beier KT, Wright M, Walsh JJ, et al. (2017): Gating of social reward by oxytocin in the ventral tegmental area. Science. 357:14061411.

21. Chaudhury D, Walsh JJ, Friedman AK, Juarez B, Ku SM, Koo JW, et al. (2013): Rapid regulation of depression-related behaviours by control of midbrain dopamine neurons. Nature. 493:532-536.

22. Grimm C, Balsters JH, Zerbi V (2021): Shedding Light on Social Reward Circuitry: (Un)common Blueprints in Humans and Rodents. Neuroscientist. 27:159183.

23. Grimm O, Nagele M, Kupper-Tetzel L, de Greck M, Plichta M, Reif A (2021): No effect of a dopaminergic modulation fMRI task by amisulpride and L-DOPA on reward anticipation in healthy volunteers. Psychopharmacology (Berl). 238:13331342.

24. Pessiglione M, Seymour B, Flandin G, Dolan RJ, Frith CD (2006): Dopaminedependent prediction errors underpin reward-seeking behaviour in humans. Nature. 442:1042-1045.

25. Swiercz AP, Iyer L, Yu Z, Edwards A, Prashant NM, Nguyen BN, et al. (2020): Evaluation of an angiotensin Type 1 receptor blocker on the reconsolidation of fear memory. Transl Psychiatry. 10:363.

26. Zhou F, Geng Y, Xin F, Li J, Feng P, Liu C, et al. (2019): Human Extinction Learning Is Accelerated by an Angiotensin Antagonist via Ventromedial Prefrontal Cortex and Its Connections With Basolateral Amygdala. Biol Psychiatry. 86:910-920. 27. Reinecke A, Browning M, Klein Breteler J, Kappelmann N, Ressler KJ, Harmer CJ, et al. (2018): Angiotensin Regulation of Amygdala Response to Threat in HighTrait-Anxiety Individuals. Biol Psychiatry Cogn Neurosci Neuroimaging. 3:826-835. 28. Marvar PJ, Goodman J, Fuchs S, Choi DC, Banerjee S, Ressler KJ (2014): Angiotensin type 1 receptor inhibition enhances the extinction of fear memory. Biol Psychiatry. 75:864-872.

29. Pulcu E, Shkreli L, Holst CG, Woud ML, Craske MG, Browning M, et al. (2019): The Effects of the Angiotensin II Receptor Antagonist Losartan on Appetitive Versus Aversive Learning: A Randomized Controlled Trial. Biol Psychiatry. 86:397-404. 
30. Chai SY, Bastias MA, Clune EF, Matsacos DJ, Mustafa T, Lee JH, et al. (2000): Distribution of angiotensin IV binding sites (AT4 receptor) in the human forebrain, midbrain and pons as visualised by in vitro receptor autoradiography. Journal of Chemical Neuroanatomy. 20:339-348.

31. Medelsohn FAO, Jenkins TA, Berkovic SF (1993): Effects of angiotensin II on dopamine and serotonin turnover in the striatum of conscious rats. Brain Research. 613:221-229.

32. Brown DC, Steward LJ, Ge J, Barnes NM (1996): Ability of angiotensin II to modulate striatal dopamine release via the AT1 receptor in vitro and in vivo. $\mathrm{Br} J$ Pharmacol. 118:414-420.

33. Narayanaswami V, Somkuwar SS, Horton DB, Cassis LA, Dwoskin LP (2013): Angiotensin AT1 and AT2 receptor antagonists modulate nicotine-evoked [(3)H]dopamine and [(3)H]norepinephrine release. Biochem Pharmacol. 86:656-665. 34. Li D, Scott L, Crambert S, Zelenin S, Eklof AC, Di Ciano L, et al. (2012): Binding of losartan to angiotensin AT1 receptors increases dopamine D1 receptor activation. J Am Soc Nephrol. 23:421-428.

35. Hosseini M, Alaei HA, Havakhah S, Neemati Karimooy HA, Gholamnezhad Z (2009): Effects of microinjection of angiotensin II and captopril to VTA on morphine self-administration in rats. Acta Biol Hung. 60:241-252.

36. Maul B, Krause W, Pankow K, Becker M, Gembardt F, Alenina N, et al. (2005): Central angiotensin II controls alcohol consumption via its AT1 receptor. FASEB J. 19:1474-1481.

37. Maes EJP, Sharpe MJ, Usypchuk AA, Lozzi M, Chang CY, Gardner MPH, et al. (2020): Causal evidence supporting the proposal that dopamine transients function as temporal difference prediction errors. Nat Neurosci. 23:176-178.

38. Steinberg EE, Keiflin R, Boivin JR, Witten IB, Deisseroth K, Janak PH (2013): A causal link between prediction errors, dopamine neurons and learning. Nat Neurosci. 16:966-973.

39. Sharpe MJ, Batchelor HM, Mueller LE, Yun Chang C, Maes EJP, Niv Y, et al. (2020): Dopamine transients do not act as model-free prediction errors during associative learning. Nat Commun. 11:106.

40. Sharpe MJ, Chang CY, Liu MA, Batchelor HM, Mueller LE, Jones JL, et al. (2017): Dopamine transients are sufficient and necessary for acquisition of modelbased associations. Nat Neurosci. 20:735-742.

41. Wake SJ, Izuma K (2017): A common neural code for social and monetary rewards in the human striatum. Soc Cogn Affect Neurosci. 12:1558-1564.

42. Izuma K, Saito DN, Sadato N (2008): Processing of social and monetary rewards in the human striatum. Neuron. 58:284-294.

43. Corlett PR, Mollick JA, Kober H Substrates of Human Prediction Error for Incentives, Perception, Cognition, and Action.

44. Lerner TN, Holloway AL, Seiler JL (2021): Dopamine, Updated: Reward Prediction Error and Beyond. Curr Opin Neurobiol. 67:123-130.

45. Zhang L, Glascher J (2020): A brain network supporting social influences in human decision-making. Sci Adv. 6:eabb4159. 
46. Birn RM, Roeber BJ, Pollak SD (2017): Early childhood stress exposure, reward pathways, and adult decision making. Proc Natl Acad Sci U S A. 114:13549-13554. 47. Bernstein DP, Stein JA, Newcomb MD, Walker E, Pogge D, Ahluvalia T, et al. (2003): Development and validation of a brief screening version of the Childhood Trauma Questionnaire. Child Abuse Negl. 27:169-190.

48. Barratt ES (1959): Anxiety and impulsiveness related to psychomotor efficiency. perceptual and motor skills.

49. Torrubia R, Ávila C, Moltó J, Caseras X (2001): The Sensitivity to Punishment and Sensitivity to Reward Questionnaire (SPSRQ) as a measure of Gray's anxiety and impulsivity dimensions. Personality and Individual Differences. 31:837-862.

50. Ohtawa M, Takayama F, Saitoh K, Yoshinaga T, Nakashima M (1993):

Pharmacokinetics and biochemical efficacy after single and multiple oral administration of losartan, an orally active nonpeptide angiotensin II receptor antagonist, in humans. Br J Clin Pharmacol. 35:290-297.

51. Sica DA, Gehr TW, Ghosh S (2005): Clinical pharmacokinetics of losartan. Clin Pharmacokinet. 44:797-814.

52. Lo MW, Goldberg MR, McCrea JB, Lu H, Furtek CI, Bjornsson TD (1995):

Pharmacokinetics of losartan, an angiotensin II receptor antagonist, and its active metabolite EXP3174 in humans. Clin Pharmacol Ther. 58:641-649.

53. Mechaeil R, Gard P, Jackson A, Rusted J (2011): Cognitive enhancement following acute losartan in normotensive young adults. Psychopharmacology (Berl). 217:51-60.

54. Culman J, von Heyer C, Piepenburg B, Rascher W, Unger T (1999): Effects of systemic treatment with irbesartan and losartan on central responses to angiotensin II in conscious, normotensive rats. Eur J Pharmacol. 367:255-265.

55. Li ZH, Bains JS, Ferguson AV (1993): Functional Evidence That the Angiotensin Antagonist Losartan Crosses the Blood-Brain-Barrier in the Rat. Brain Research Bulletin. 30:33-39.

56. Spielberger C, Goruch R, Lushene R, Vagg P, Jacobs G (1983): Manual for the state-trait inventory STAI (form Y). Mind Garden, Palo Alto, CA, USA.

57. Watson D, Clark LA, Tellegen A (1988): Development and validation of brief measures of positive and negative affect: the PANAS scales. J Pers Soc Psychol. 54:1063-1070.

58. Esteban O, Markiewicz CJ, Blair RW, Moodie CA, Isik AI, Erramuzpe A, et al. (2019): fMRIPrep: a robust preprocessing pipeline for functional MRI. Nat Methods. 16:111-116.

59. Friston KJ, Jezzard P, Turner R (1994): Analysis of Functional MRI Time-Series Human Brain Mapping.

60. Faulkner ML, Momenan R, Leggio L (2021): A neuroimaging investigation into the role of peripheral metabolic biomarkers in the anticipation of reward in alcohol use. Drug Alcohol Depend. 221:108638.

61. Lawn W, Hill J, Hindocha C, Yim J, Yamamori Y, Jones G, et al. (2020): The acute effects of cannabidiol on the neural correlates of reward anticipation and feedback in healthy volunteers. J Psychopharmacol. 34:969-980. 
62. Rademacher L, Krach S, Kohls G, Irmak A, Grunder G, Spreckelmeyer KN (2010): Dissociation of neural networks for anticipation and consumption of monetary and social rewards. Neuroimage. 49:3276-3285.

63. Zhou X, Zimmermann K, Xin F, Zhao W, Derckx RT, Sassmannshausen A, et al. (2019): Cue Reactivity in the Ventral Striatum Characterizes Heavy Cannabis Use, Whereas Reactivity in the Dorsal Striatum Mediates Dependent Use. Biol Psychiatry Cogn Neurosci Neuroimaging. 4:751-762.

64. Trutti AC, Fontanesi L, Mulder MJ, Bazin PL, Hommel B, Forstmann BU (2021): A probabilistic atlas of the human ventral tegmental area (VTA) based on 7 Tesla MRI data. Brain Struct Funct. 226:1155-1167.

65. McLaren DG, Ries ML, Xu G, Johnson SC (2012): A generalized form of context-dependent psychophysiological interactions (gPPI): a comparison to standard approaches. Neuroimage. 61:1277-1286.

66. Volman I, Pringle A, Verhagen L, Browning M, Cowen PJ, Harmer CJ (2021): Lithium modulates striatal reward anticipation and prediction error coding in healthy volunteers. Neuropsychopharmacology. 46:386-393.

67. Sambrook TD, Goslin J (2016): Principal components analysis of reward prediction errors in a reinforcement learning task. Neuroimage. 124:276-286.

68. Rescorla RA, Wagner AR (1972): A theory of Pavlovian conditioning: Variations in the effectiveness of reinforcement and nonreinforcement. In: Black H, Prokasy WF, editors. Classical conditioning II: Current research and theory. New York: AppletonCentury-Crofts.

69. Cao Z, Bennett M, Orr C, Icke I, Banaschewski T, Barker GJ, et al. (2019):

Mapping adolescent reward anticipation, receipt, and prediction error during the monetary incentive delay task. Hum Brain Mapp. 40:262-283.

70. Glascher JP, O'Doherty JP (2010): Model-based approaches to neuroimaging: combining reinforcement learning theory with fMRI data. Wiley Interdiscip Rev Cogn Sci. 1:501-510.

71. Metereau E, Dreher JC (2013): Cerebral correlates of salient prediction error for different rewards and punishments. Cereb Cortex. 23:477-487.

72. Slotnick SD (2017): Cluster success: fMRI inferences for spatial extent have acceptable false-positive rates. Cogn Neurosci. 8:150-155.

73. Murugan M, Jang HJ, Park M, Miller EM, Cox J, Taliaferro JP, et al. (2017): Combined Social and Spatial Coding in a Descending Projection from the Prefrontal Cortex. Cell. 171:1663-1677 e1616.

74. Modi ME, Sahin M (2019): A unified circuit for social behavior. Neurobiol Learn Mem. 165:106920.

75. Gordon I, Jack A, Pretzsch CM, Vander Wyk B, Leckman JF, Feldman R, et al. (2016): Intranasal Oxytocin Enhances Connectivity in the Neural Circuitry Supporting Social Motivation and Social Perception in Children with Autism. Sci Rep. 6:35054.

76. Greene RK, Spanos M, Alderman C, Walsh E, Bizzell J, Mosner MG, et al. (2018): The effects of intranasal oxytocin on reward circuitry responses in children with autism spectrum disorder. J Neurodev Disord. 10:12. 
77. Averbeck BB, Costa VD (2017): Motivational neural circuits underlying reinforcement learning. Nat Neurosci. 20:505-512.

78. Uddin LQ (2015): Salience processing and insular cortical function and dysfunction. Nat Rev Neurosci. 16:55-61.

79. Zhou F, Li J, Zhao W, Xu L, Zheng X, Fu M, et al. (2020): Empathic pain evoked by sensory and emotional-communicative cues share common and process-specific neural representations. Elife. 9.

80. Dohmatob E, Dumas G, Bzdok D (2020): Dark control: The default mode network as a reinforcement learning agent. Hum Brain Mapp. 41:3318-3341.

81. Gueguen MCM, Lopez-Persem A, Billeke P, Lachaux JP, Rheims S, Kahane P, et al. (2021): Anatomical dissociation of intracerebral signals for reward and punishment prediction errors in humans. Nat Commun. 12:3344.

82. Keren H, Chen G, Benson B, Ernst M, Leibenluft E, Fox NA, et al. (2018): Is the encoding of Reward Prediction Error reliable during development? Neuroimage.

178:266-276.

83. Suzuki S, Lawlor VM, Cooper JA, Arulpragasam AR, Treadway MT (2021): Distinct regions of the striatum underlying effort, movement initiation and effort discounting. Nat Hum Behav. 5:378-388.

84. Gordon EM, Laumann TO, Marek S, Newbold DJ, Hampton JM, Seider NA, et al. (2021): Human Fronto-Striatal Connectivity is Organized into Discrete Functional Subnetworks.

85. Hetu S, Luo Y, D'Ardenne K, Lohrenz T, Montague PR (2017): Human substantia nigra and ventral tegmental area involvement in computing social error signals during the ultimatum game. Soc Cogn Affect Neurosci. 12:1972-1982. 